\title{
2020 international practice patterns in adult joint reconstruction surgery: a survey of members of the International Society of Orthopaedic Centers
}

\author{
Daniel C. Santana ${ }^{1} \cdot$ Alejandro Gonzalez Della Valle ${ }^{2} \cdot$ Alison K. Klika $^{1} \cdot$ Briana Bloom $^{2} \cdot$ Mathias Bostrom $^{2}$. \\ Nicolas S. Piuzzi ${ }^{1}$ (1)
}

Received: 4 January 2021 / Accepted: 12 January 2021 / Published online: 22 January 2021

(c) The Author(s), under exclusive licence to Springer-Verlag France SAS part of Springer Nature 2021

\begin{abstract}
Purpose It is important to assess global trends in the practice of adult reconstruction orthopaedic surgery to understand how new evidence is being implemented. The International Society of Orthopaedic Centers (ISOC) is a consortium of academic orthopaedic centers whose members' practices likely reflect contemporary evidence and indicate how orthopaedic surgery residents and fellows are trained.

Methods We administered a 65 question, electronic survey of adult reconstruction surgeons across the ISOC centers in September 2020 to assess practice patterns. Results were assessed using descriptive statistics or by modeling the underlying response distribution, and the analysis was stratified by hospital region.

Results 79 surgeons across 19 ISOC centers in 5 continents (Asia, Australia, Europe, North America, South America) completed the survey. Selected findings include: in total hip arthroplasty (THA), the posterolateral approach was used for $71 \pm 42 \%$ of THA (mean \pm standard deviation) and the direct anterior approach in $18 \% \pm 34 \%$. In total knee arthroplasty, posterior-stabilized $(66 \% \pm 39 \%)$ and cruciate-retaining $(19 \pm 33 \%)$ implants were most common. Robots were available in $56 \%$ (44 of 79) of surgeons' centers more commonly in Asia, Australia, and North America. Tranexamic acid was routinely used in arthroplasty by 99\% (78 of 79) of surgeons. Eighty-six percent (68 of 79) submit data to joint or other registries. Virtual visits were used for $13 \% \pm 16 \%$ of outpatient visits and by $82 \%$ (64 of 79) of surgeons overall.

Conclusions These findings may be of use now for surgeons to consider the practices of their peers at high-volume academic institutions, and in the future as we track temporal trends.
\end{abstract}

Keywords Arthroplasty · Practice survey · Implant selection · Surgical approach · Virtual visit

Supplementary Information The online version contains supplementary material available at https://doi.org/10.1007/s0059 0-021-02876-7.

Nicolas S. Piuzzi

piuzzin@ccf.org

1 Department of Orthopaedic Surgery, Orthopaedic and Rheumatologic Institute, Cleveland Clinic, Cleveland, $\mathrm{OH} 44195$, USA

2 Department of Orthopaedic Surgery, Hospital for Special Surgery, New York, NY, USA

\section{Introduction}

Adult reconstruction orthopaedic surgeons perform over 1-million hip and knee replacements annually in the U.S. alone, with a growing volume [1]. Surgical practice constantly evolves in response to new research and technology, economic factors, and patient characteristics. In this changing landscape, it is important to track surgical practices to understand how new findings are being applied [2]. Documentation of current practices allows surgeons to understand the practice patterns of their peers and to reflect on their own. Further, analysis of global trends in reconstruction surgery may offer insight into factors driving practice variability. The purpose of this study was to assess the current state of adult reconstruction surgical practice globally. These 
results may be compared with future surveys to track trends in global surgical practice over time.

\section{Materials and methods}

In September 2020, we surveyed the International Society of Orthopaedic Centers (ISOC), an international consortium of 23 academic orthopaedic centers [3]. To qualify for ISOC membership, institutions must demonstrate significant research output in orthopaedics, perform at least 5000 orthopaedic procedures per year, have at least 20 staff orthopaedic surgeons, and train orthopaedic residents or fellows.

The survey was based upon a survey administered by the American Association of Hip and Knee Surgeons (AAHKS) [4] and contained 65 multiple-choice or yes-no questions. We used multiple-choice questions for numeric quantities by creating binned responses in order to facilitate survey completion and reduce respondent bias in providing estimates [5].

The survey was conducted electronically using Google Forms and disseminated to adult reconstruction surgeons via email through the heads of department in each ISOC center. This study was deemed exempt by the Cleveland Clinic Institutional Review Board.

\section{Data analysis}

The results were assessed using descriptive statistics. For binned data, a probability distribution was fitted using maximum-likelihood estimation in order to estimate summary statistics of the data instead of describing the bin frequencies, which would be unwieldy to describe and interpret [6]. After assessing each variable's distribution independently, beta or gamma distributions were utilized. These are both two-parameter distributions that are very flexible [7], making them well suited to the bimodal and skewed response distributions we observed.

Responses were stratified by region to understand how location may affect practice characteristics. To identify potential differences, in practice across regions, we performed Fisher exact tests (categorical data) and Kruskal-Wallis tests (binned data). We corrected for multiple comparisons using the Benjamini-Hochberg procedure with a false discovery rate of 0.1 , which ultimately classified results with $p$ values less than 0.025 as statistically significant. All analysis was performed with Python 3.8.5.

\section{Results}

The full survey results and analysis may be found in Appendices A-E. Seventy-nine surgeons across 19 ISOC centers responded to the survey (Fig. 1), with 28 responses from

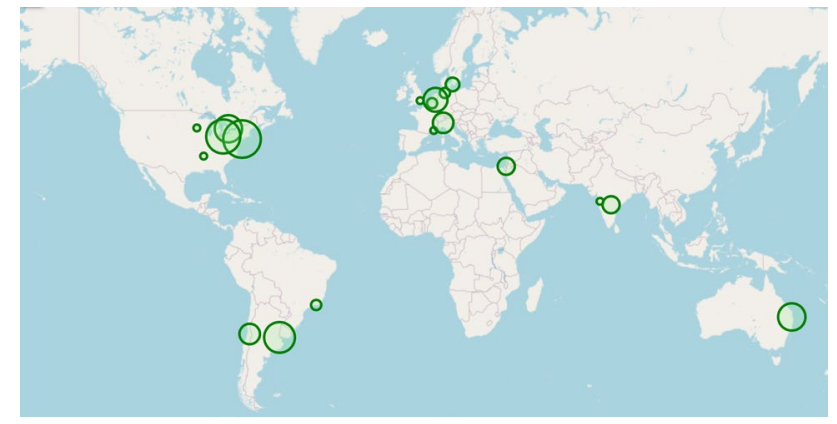

Fig. 1 ISOC provider responses by geographic location. The size of each marker corresponds to the number of responses received at that location

North America (N.A.), 20 from Europe (Eur.), 15 from South America (S.A.), 9 from Asia, and 7 from Australia. We grouped Asia and Australia (A\&A) for the regional analysis due to fewer responses. The average surgeon was $48 \pm 13$ years old (mean \pm standard deviation) and had been in practice for $17 \pm 14$ years. Almost all respondents were fellowship trained $(95 \%, 75 / 79)$, with the lowest rate of fellowship training in Eur. $(85 \%, 17 / 20 ; p=0.020)$.

Primary total joint arthroplasty (TJA) accounted for $67 \pm 20 \%$ and revision TJA for $18 \pm 15 \%$ of respondents' surgical practices. Ambulatory TJA was performed in $9 \pm 19 \%$ of TJA overall, but 59\% (47/79) of surgeons never performed ambulatory TJA. The use of ambulatory TJA varied by region with $79 \%(22 / 28)$ of surgeons in N.A. performing at least $1 \%$ of their TJA in the ambulatory setting $(p<0.001)$.

A robot for TJA was available in 56\% (44/79) of respondents' surgical centers, but it was significantly more common in A\&A $(94 \%, 15 / 16)$ and N.A. $(75 \%, 21 / 28)$ than S.A. $(33 \%, 5 / 15)$ and Eur. $(15 \%, 3 / 20 ; p<0.001)$.

Surgeons most commonly used 1 dose of preoperative antibiotics $(75 \%, 59 / 79)$, but surgeons in Eur. and N.A. were more likely to use 3 doses compared to other regions [20\% $(4 / 20)$ and $18 \%(5 / 28)$, respectively; $p<0.001]$.

Tranexamic acid (TXA) was used by $99 \%$ (78/79) of surgeons in primary TJA when there was no contraindication, with intravenous (IV) TXA most common (52\%, 41/79), followed by a combination of delivery methods (42\%, 33/79), and topical TXA only $(6 \%, 5 / 79)$. Topical TXA alone was more common in A\&A $(19 \%, 3 / 16)$ compared to S.A. (0\%), Eur. $(5 \%, 1 / 20)$ and N.A. $(4 \%, 1 / 28 ; p<0.001)$.

Surgical cutoffs using body mass index (BMI) and hemoglobin A1c (HbA1c) were used by 37\% (29/79) and 63\% (50/79) of surgeons, respectively. The most frequent cutoff for BMI was greater than $40 \mathrm{~kg} / \mathrm{m}^{2}(52 \%, 15 / 29)$, and for HbA1c, greater than $8.0 \%(52 \%, 26 / 50)$. Regionally, the most common cutoffs for BMI differed $(p<0.001)$ with $35 \mathrm{~kg} / \mathrm{m}^{2}$ most common in S.A. $(50 \%, 3 / 6), 45 \mathrm{~kg} / \mathrm{m}^{2}$ in A\&A $(50 \%, 3 / 6)$, and $40 \mathrm{~kg} / \mathrm{m}^{2}$ in Eur. $(89 \%, 8 / 9)$ and N.A. 
$(56 \%, 5 / 9)$. Only $20 \%$ (4/20) of providers in Eur. used cutoffs for $\mathrm{HbA} 1 \mathrm{c}$, while in N.A. a HbA1c cutoff was used by $93 \%$ $(26 / 28 ; p<0.001)$.

\section{Total hip arthroplasty practice}

The average surgeon performed $135 \pm 115$ total hip arthroplasties (THA) annually, with the highest volume in N.A. $(p=0.006)$. In centers where a robot was available, $13 \pm 25 \%$ of THA was performed robotically, but most surgeons with a robot available did not utilize it (median 0\%, IQR 0-9\%).

Cemented stems were used in an average of $20 \% \pm 21 \%$ of THA and cemented cups in $6 \pm 16 \%$ with a distribution
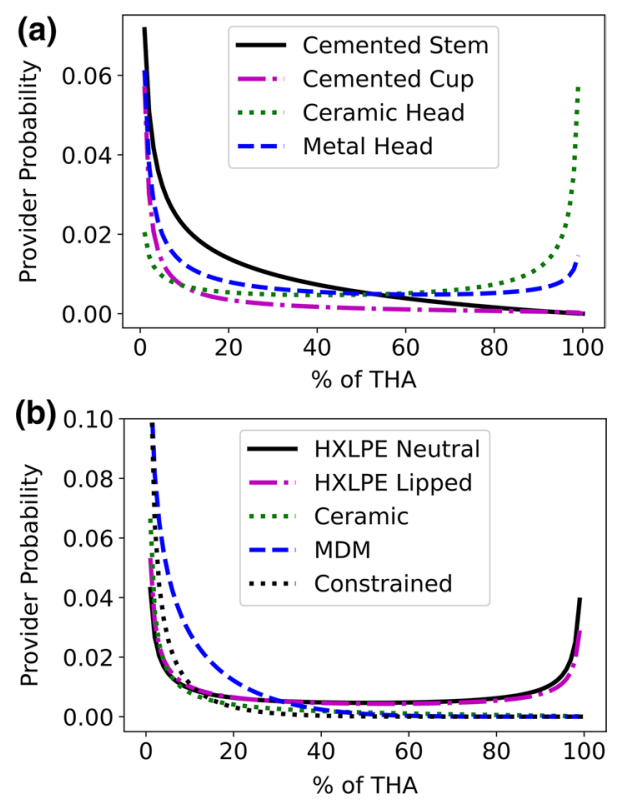

Fig. 2 Implant choice in total hip arthroplasty (THA). Plots represent the probability of surgeons using a given implant (a) or liner (b) in a certain percentage of their THA cases. Bimodal distributions indicate that some providers nearly always use that implant type while other providers rarely do. MDM modular dual-mobility liner indicating than many providers never use a cemented THA (Fig. 2a; Table 1). A fully cemented THA was more common in Eur. than other regions ( $p=0.003$ for cemented stems and $p=0.018$ for cemented cups). A ceramic head was used in $65 \pm 36 \%$ of THA and a metal head in $32 \pm 34 \%$. The most commonly used liners for THA were highly cross-linked polyethylene neutral (HXLPE; $49 \pm 39 \%$ ), followed by HXLPE-lipped $(40 \pm 38 \%)$, modular dual-mobility (MDM; $9 \pm 11 \%)$, ceramic $(6 \pm 15 \%)$, and constrained $(3 \pm 6 \%$; Fig. 2b; Table 1). Distributions of implant choice were generally bimodal, indicating marked individual preferences for or against each. HXLPE-lipped liners were more commonly used by providers in S.A. and A\&A compared to the other regions $(p<0.001)$, while in Eur. and N.A. HXLPE-neutral liners were preferred $(p<0.001)$. Ceramic liners were used more frequently in A\&A and Eur. compared to the other regions $(p<0.001)$.

The posterolateral approach for THA was used $71 \pm 42 \%$ of the time (Fig. 3; Table 2). The next most common approaches were direct anterior used in $18 \pm 34 \%$ of THA and direct lateral in $11 \pm 26 \%$ of THA. The anterolateral approach was rarely used (mean $4 \pm 13 \%$ ). Approaches demonstrated bimodal distributions indicating individual surgeon preferences for or against each.

Spinal anesthesia was used in $83 \pm 28 \%$ of THA and general anesthesia in $20 \pm 24 \%$ (Fig. 4a; Table 3). Local or periarticular injections for analgesia were used in $47 \pm 45 \%$.

\section{Total knee arthroplasty practice}

The average respondent performed $147 \pm 136$ total knee arthroplasties (TKA) annually. Patient specific instrumentation (PSI) was infrequently used in TKA (mean $8 \pm 21 \%$ ) with most providers never using PSI (median 0\%). Unicompartmental knee arthroplasty (UKA) was performed by $62 \%$ (49/79) of surgeons. When UKA was performed, $92 \pm 13 \%$ of cases were medial compartmental, $3 \pm 5 \%$ were lateral compartmental, and $2 \pm 10 \%$ were patellofemoral. In centers
Table 1 Implant choice in total hip arthroplasty

\begin{tabular}{lllccc}
\hline Implant & Mean (\%) & $\begin{array}{l}\text { Standard devia- } \\
\text { tion }(\%)\end{array}$ & Median $(\%)$ & $\begin{array}{l}\text { 25th percen- } \\
\text { tile }(\%)\end{array}$ & $\begin{array}{l}\text { 75th } \\
\text { percentile } \\
(\%)\end{array}$ \\
\hline Non-cemented stem & 80 & 21 & 97 & 87 & 69 \\
Non-cemented cup & 94 & 16 & 100 & 100 & 98 \\
Ceramic head & 65 & 36 & 31 & 79 & 98 \\
Metal head & 32 & 34 & 2 & 18 & 61 \\
HXLPE-neutral liner & 49 & 39 & 47 & 7 & 91 \\
HXLPE-lipped liner & 40 & 38 & 26 & 2 & 80 \\
Ceramic liner & 6 & 15 & 0 & 0 & 3 \\
MDM liner & 9 & 11 & 5 & 1 & 13 \\
Constrained liner & 3 & 6 & 0 & 0 & 3 \\
\hline
\end{tabular}




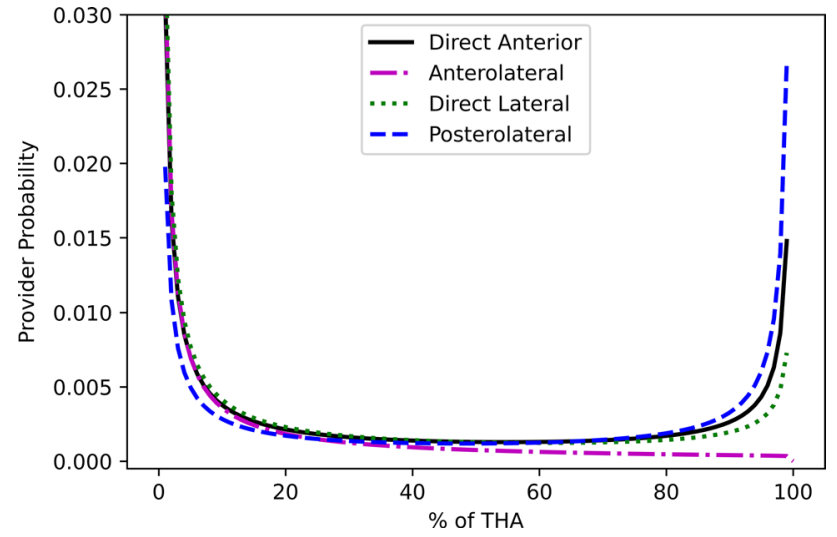

Fig. 3 Surgical approach in total hip arthroplasty (THA). Plot represents the probability of a surgeon choosing a given approach in a certain percentage of their THA cases. Bimodal distributions indicate that some providers nearly always use that approach while other providers rarely do

where a robot was available, $20 \pm 29 \%$ of TKA and $44 \pm 45 \%$ of UKA were performed robotically, but most surgeons with a robot available rarely used it, while a minority frequently did (median $4 \%$ for TKA and $20 \%$ for UKA).

Cementless implants were only used in $14 \pm 29 \%$ of primary TKA. The most common primary TKA implants were posterior-stabilized (PS; $66 \pm 39 \%$ ) and cruciate retaining $(\mathrm{CR} ; 19 \pm 33 \%)$, followed by ultra-congruent (UC; $13 \pm 28 \%)$, other inserts $(9 \pm 21 \%)$, and varus-valgus constrained (4 $4 \%$; Fig. 5; Table 4). Implant and liner choice were generally bimodal, again indicating strong individual preference for or against each.

Tourniquets were used in an average of $67 \pm 45 \%$ of TKA with the majority of providers opting to use them nearly always, while a minority rarely did so (median 100\%, IQR $1-100 \%)$.

Spinal anesthesia was used in $81 \pm 28 \%$ of TKA and general anesthesia in $18 \pm 20 \%$ (Fig. 4b; Table 3). Providers in S.A. were least likely to use general anesthesia $(p=0.008)$. Regional nerve blocks were used in $61 \pm 42 \%$ of TKA, more commonly in N.A. $(p<0.001)$, and local or periarticular injections for analgesia were used in $82 \pm 34 \%$ of TKA.

\section{Compensation and structural elements of practice}

Providers were most commonly compensated by fixed salary $(37 \%, 29 / 79)$ followed by fee-for-service $(33 \%, 26 / 79)$ and salary with performance incentives $(22 \%, 17 / 79)$. No providers were compensated solely by a relative value unit (RVU)-based scheme. Fee-for-service compensation was more common in S.A. (53\%, 8/15), A\&A (38\%, 6/16), and N.A. $(43 \%, 12 / 28)$, but fixed salary more common in Eur. $(85 \%, 17 / 20 ; p<0.001)$.

Consulting for orthopaedic or pharmaceutical companies was performed by $58 \%$ (46/79) of providers, with the highest rate in N.A. $(79 \%, 22 / 28 ; p=0.020)$ Fewer providers received royalties from an orthopaedic manufacturer $(23 \%$, 18/79). Consulting income varied widely, but it was most commonly between $\$ 5000-\$ 20,000$ (23\%, 18/79; U.S. dollars).
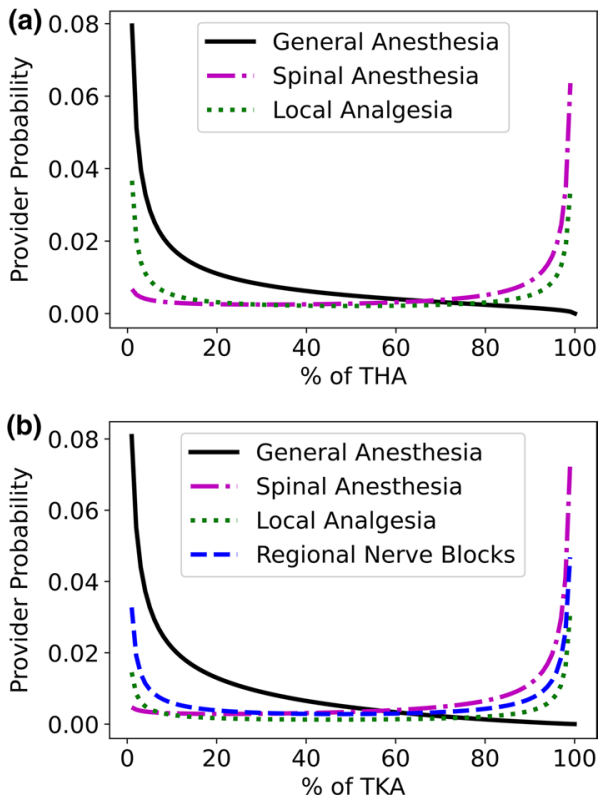

Fig. 4 Use of various anesthesia or analgesia in total hip arthroplasty (THA) and total knee arthroplasty (TKA). Plots represent the probability of a surgeon choosing a given form of anesthesia or analgesia in a certain percentage of their THA (a) or TKA (b) cases. Bimodal distributions indicate that some providers nearly always use that form of anesthesia or analgesia while other providers rarely do
Table 2 Surgical approach in total hip arthroplasty

\begin{tabular}{lclccc}
\hline Approach & Mean (\%) & $\begin{array}{l}\text { Standard devia- } \\
\text { tion }(\%)\end{array}$ & Median $(\%)$ & $\begin{array}{l}\text { 25th percentile } \\
(\%)\end{array}$ & $\begin{array}{l}\text { 75th } \\
\text { percentile } \\
(\%)\end{array}$ \\
\hline Direct anterior & 18 & 34 & 0 & 0 & 11 \\
Anterolateral & 4 & 13 & 0 & 0 & 0 \\
Direct lateral & 11 & 26 & 0 & 0 & 2 \\
Posterolateral & 71 & 42 & 100 & 20 & 100 \\
\hline
\end{tabular}


Table 3 Use of various anesthesia or analgesia in total hip arthroplasty (THA) and total knee arthroplasty (TKA)

\begin{tabular}{llllcr}
\hline & Mean (\%) & $\begin{array}{l}\text { Standard devia- } \\
\text { tion }(\%)\end{array}$ & Median (\%) & $\begin{array}{l}\text { 25th percen- } \\
\text { tile }(\%)\end{array}$ & $\begin{array}{l}\text { 75th } \\
\text { percentile } \\
(\%)\end{array}$ \\
\hline THA anesthesia/analgesia & & & & & \\
General anesthesia & 20 & 24 & 10 & 1 & 32 \\
Spinal anesthesia & 83 & 28 & 99 & 79 & 100 \\
Local analgesia & 47 & 45 & 34 & 0 & 99 \\
TKA anesthesia/analgesia & & & & 2 & 28 \\
General anesthesia & 18 & 20 & 10 & 73 & 100 \\
Spinal anesthesia & 81 & 28 & 97 & 91 & 100 \\
Local analgesia & 82 & 34 & 100 & 10 & 100 \\
Regional nerve blocks & 61 & 42 & 83 & & \\
\hline
\end{tabular}

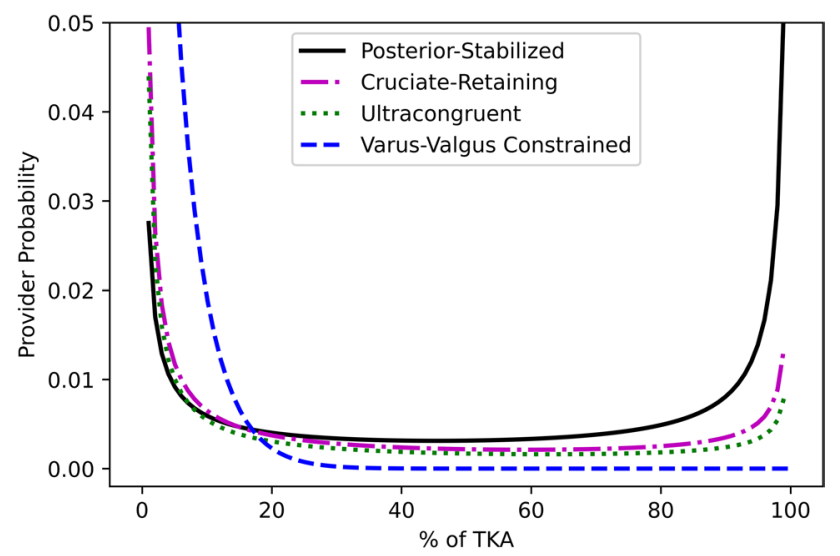

Fig. 5 Implant choice in total knee arthroplasty (TKA). Plot represents the probability of a surgeon choosing a given implant type in a certain percentage of their TKA cases. Bimodal distributions indicate that some providers nearly always use that implant type while other providers rarely do

The average amount of practice call taken per month was $7 \pm 6$ days, with the lowest amount in N.A. where $93 \%(26 / 28)$ took 5 days or fewer $(p=0.019)$. The average amount of emergency room call was $2 \pm 3$ days per month.

Virtual visits were used for $13 \pm 16 \%$ of outpatient visits and by $82 \%$ (64/79) of surgeons overall.

Electronic medical records (EMR) were widely utilized by providers, with $56 \%$ (44/79) reporting a completely paperless office and 38\% (30/79) with an EMR but still using some charts or paper. EMRs were most commonly funded by the hospital $(67 \%, 49 / 73)$, by hospital and practice revenue $(16 \%, 12 / 73)$, or by the practice alone $(12 \%, 9 / 73)$. Providers in Eur. were least likely to have an EMR $(20 \%, 4 / 20$, $p=0.004$ ).

Staff members in respondents' practices, such as physician extenders, were most commonly funded by the hospital $(53 \%, 42 / 79)$, followed by the hospital and practice group together $(20 \%, 16 / 79$ or by individual physicians $(20 \%$, 16/79). Significantly more physicians employed their own staff in A\&A $(44 \%, 7 / 16)$ and N.A. $(32 \%, 9 / 28)$ than S.A. $(0 \%)$ and Eur. $(0 \% ; p<0.001)$.

Overall, $86 \%$ (68/79) of respondents submit data to joint or other registries. Registry participation was significantly less common in S.A. (40\%, 6/15) compared to A\&A (94\%, $15 / 16)$, Eur. (100\%, 20/20), and N.A. $(96 \%, 27 / 28)$ where it was nearly universal $(p<0.001)$ (Table 5$)$.

\section{Discussion}

In this survey, we assessed the practice patterns of 79 international joint reconstruction surgeons who were part of the International Society of Orthopaedic Centers (ISOC) representing surgeons from high-volume academic centers.

As expected, respondents' surgical practices were heavily composed of primary and revision total joint arthroplasty performing hundreds of joint replacements annually,
Table 4 Implant choice in total knee arthroplasty

\begin{tabular}{lclccc}
\hline Implant & Mean (\%) & $\begin{array}{l}\text { Standard } \\
\text { deviation }(\%)\end{array}$ & Median (\%) & $\begin{array}{l}\text { 25th percen- } \\
\text { tile }(\%)\end{array}$ & $\begin{array}{l}\text { 75th } \\
\text { percentile } \\
(\%)\end{array}$ \\
\hline Posterior-stabilized & 66 & 39 & 89 & 24 & 100 \\
Cruciate-retaining & 19 & 33 & 0 & 0 & 24 \\
Ultracongruent & 13 & 28 & 0 & 0 & 6 \\
Varus-valgus constrained & 4 & 4 & 2 & 1 & 5 \\
\hline
\end{tabular}


Table 5 Tourniquet use in total knee arthroplasty stratified by region

\begin{tabular}{lllllc}
\hline Region & $0 \%$ & $0-25 \%$ & $25-50 \%$ & $50-75 \%$ & $75-100 \%$ \\
\hline North America & 1 & 1 & 0 & 0 & 26 \\
Europe & 8 & 3 & 0 & 0 & 6 \\
Asia and Australia & 5 & 0 & 0 & 0 & 10 \\
South America & 3 & 1 & 1 & 0 & 2 \\
\hline
\end{tabular}

similar to the results of the AAHKS 2019 survey [4]. This suggests that orthopaedic surgeons outside the US have similar practice patterns in this regard.

Robots for robotic TJA were generally available in N.A., and A\&A, but less so in S.A. or Eur. In the AAHKS survey, $39 \%$ of respondents had robots available, compared to $56 \%$ overall and $75 \%$ in N.A. in this survey.

There was good agreement on some elements of arthroplasty practice: three out of four surgeons give one dose of preoperative antibiotics, and the use of tranexamic acid was nearly universal if there was no contraindication. Regarding surgical cutoffs, there was more surgeon variability. Approximately 2 in 5 used a BMI cutoff and 3 in 5 used a HbA1c cutoff, with cutoffs of $40 \mathrm{~kg} / \mathrm{m}^{2}$ for BMI and $8.0 \%$ for $\mathrm{HbA} 1 \mathrm{c}$ most common.

Spinal anesthesia was used for the majority (> 80\%) of THA and TKA, with general anesthesia likely reserved for more complex cases. This rate is much higher than observed in a U.S. study of anesthesia use in TKA between 2010 and 2013, where spinal anesthesia was used in only $31 \%$ of cases [8]. We cannot be certain whether this difference reflects temporal trends or a difference in practice patterns of respondents to our survey.

\section{THA implants and approaches}

It is evident from this survey that providers have strong implant preferences for both THA and TKA and tend not to vary between different implants. For THA, the use of cemented implants was not common overall, but they were more common in Europe than other regions. An important implication of regional differences in implant use is that residents or fellows training in different regions do not receive the same experience with implants.

Surgical approach for THA was dominated by the posterolateral approach, though as reflected in many other decisions, some providers rarely used this approach and instead opted for the direct anterior or direct lateral approaches. Compared to a 2013 survey in Eur. and N.A., respondents to our survey favored the posterolateral approach far more and the direct lateral approach far less (2013 survey: posterolateral $45 \%$, direct lateral $42 \%$, anterior $10 \%$ ) [9].

\section{TKA implants and tourniquets}

Overall, TKA cementless implants were infrequently used, consistent with a study of the Nordic arthroplasty registry [10]. The most popular insert choice was posterior-stabilized (66\%), which differs from a recent systematic review that observed a fairly even split between posterior-stabilized and cruciate-retaining implant use [11]. Tourniquet use was common, particularly in N.A., consistent with growing evidence from randomized trials that tourniquets are safe and effective in TKA [12].

\section{Compensation and structural elements of practice}

Approximately 1 in 5 surgeons overall received some form of performance incentives, but no surgeons were compensated solely by a RVU scheme, which differs from the AAHKS 2019 survey where $19 \%$ of respondents were compensated by RVUs [4]. More surgeons in our survey also received consulting income $(58 \%)$ or royalties $(23 \%)$ compared to AAHKS 2019 respondents (35\% and 12\%, respectively) [4].

A majority of respondents (82\%) used virtual visits for outpatient appointments, though the number of visits performed virtually was low (13\%). This survey was administered in the midst of the COVID-19 pandemic. As such, we suspect that the use of virtual visits is higher than it was previously, as was found in a survey administered in April 2020 where the use of virtual visits increased to $48 \%$ from $18 \%$ in 2018 [13].

We found that the majority of surgeons $(86 \%)$ submit data to joint registries or other registries, compared to $50 \%$ in the AAHKS 2019 survey, but in South America only a minority did so. This may in part be because of a lack of national joint registries in South American countries [14].

\section{Limitations}

We surveyed the practice patterns of academic surgeons at centers that are a part of ISOC, which limits the generalizability of these findings particularly beyond academic orthopaedic practice. Some findings may represent the practices of few surgeons and should be interpreted in light of this. There is likely measurement bias in the results as it may be difficult for respondents to estimate the frequency at which they employ certain practices, though we mitigated this bias through multiple-choice questions and the analysis methods.

\section{Conclusions}

In this international survey of adult reconstruction orthopaedic surgeons, we examined the current state of surgical practice and uncovered regional trends. These findings may 
be of use now for surgeons to consider the practices of their peers, and in the future as we track temporal trends.

Author contributions DCS and AGDV wrote the manuscript, DCS and AK performed the analysis, all authors designed the survey, $\mathrm{BB}, \mathrm{MB}$ and NSP reviewed the manuscript critically.

Funding No funding was received to perform this investigation.

Availability of data and materials Aggregated data are available within the included appendices.

Code availability Code may be made available upon request.

\section{Compliance with ethical standards}

Conflict of interest BB and MB are officers on the International Society of Orthopaedics board of directors.

Ethical approval This study was deemed exempt by the Cleveland Clinic Institutional Review Board.

Consent to participate Subjects were provided consent documentation in the email containing the survey. Consent was implied through voluntary participation in the survey.

Consent to publish Not applicable.

\section{References}

1. Sloan M, Premkumar A, Sheth NP (2018) Projected volume of primary total joint arthroplasty in the U.S., 2014 to 2030 . J Bone Jt Surg Am 100:1455-1460. https://doi.org/10.2106/JBJS.17.01617

2. Emara AK, Klika AK, Piuzzi NS (2020) Evidence-based orthopedic surgery—-from synthesis to practice. JAMA Surg 155:10091010. https://doi.org/10.1001/jamasurg.2020.1521

3. International Society of Orthopaedic Centers, Ltd. https://www. isocweb.org

4. Lieberman JR, Browne JA, Bolognesi MP (2020) Practice management strategies among current members of the american association of hip and knee surgeons. J Arthroplasty 35:S42-S45. https://doi.org/10.1016/j.arth.2020.01.066
5. Angelov AG, Ekström M (2019) Maximum likelihood estimation for survey data with informative interval censoring. AStA Adv Stat Anal 103:217-236. https://doi.org/10.1007/s10182-01800329-X

6. Gentleman R, Geyer CJ (1994) Maximum likelihood for interval censored data: consistency and computation. Biometrika $81: 618-623$

7. Mun J (2008) Understanding and choosing the right probability distributions. In: Advanced Analytical Models: Over 800 Models and 300 Applications from the Basel II Accord to Wall Street and Beyond. pp 899-917

8. Fleischut PM, Eskreis-Winkler JM, Gaber-Baylis LK et al (2015) Variability in anesthetic care for total knee arthroplasty: an analysis from the anesthesia quality institute. Am J Med Qual 30:172179. https://doi.org/10.1177/1062860614525989

9. Chechik O, Khashan M, Lador R et al (2013) Surgical approach and prosthesis fixation in hip arthroplasty world wide. Arch Orthop Trauma Surg 133:1595-1600. https://doi.org/10.1007/ s00402-013-1828-0

10. Niemeläinen MJ, Mäkelä KT, Robertsson O et al (2020) The effect of fixation type on the survivorship of contemporary total knee arthroplasty in patients younger than 65 years of age: a registerbased study of 115,177 knees in the Nordic Arthroplasty Register Association (NARA) 2000-2016. Acta Orthop 91:184-190. https ://doi.org/10.1080/17453674.2019.1710373

11. Longo UG, Ciuffreda M, Mannering N et al (2018) Outcomes of posterior-stabilized compared with cruciate-retaining total knee arthroplasty. J Knee Surg 31:321-340. https://doi. org/10.1055/s-0037-1603902

12. Goel R, Rondon AJ, Sydnor K et al (2019) Tourniquet use does not affect functional outcomes or pain after total knee arthroplasty: a prospective, double-blinded, randomized controlled trial. J Bone Jt Surg Am 101:1821-1828. https://doi.org/10.2106/ JBJS. 19.00146

13. Hawkins M (2020) Physicians and COVID-19: a survey examining how physicians are being affected by and are responding to the coronavirus pandemic. Dallas, TX

14. Hughes RE, Batra A, Hallstrom BR (2017) Arthroplasty registries around the world: valuable sources of hip implant revision risk data. Curr Rev Musculoskelet Med 10:240-252. https://doi. org/10.1007/s12178-017-9408-5

Publisher's Note Springer Nature remains neutral with regard to jurisdictional claims in published maps and institutional affiliations. 